\title{
Neutron-Induced Single Events in a COTS Soft-Error Free SRAM at Low Bias Voltage
}

\author{
Juan A. Clemente, Francisco J. Franco, Francesca Villa, Maud Baylac, Pablo Ramos, Vanessa Vargas, \\ Hortensia Mecha, Juan A. Agapito, and Raoul Velazco
}

\begin{abstract}
This paper presents an experimental study of the sensitivity to $15-\mathrm{MeV}$ neutrons at low bias voltage of advanced low-power SRAMs by Renesas Electronics. The most interesting results are the occurrence of clusters of bitflips, hard errors only visible at low voltage, appearing along with single event upsets. The physical mechanisms are briefly discussed.
\end{abstract}

Index Terms-COTS, LPSRAM, neutron tests, radiation hardness, reliability, soft error, SRAM

\section{INTRODUCTION}

M ODERN electronic systems are designed to minimize power consumption. In standby, non-critical devices can be switched off, only keeping connected the main parts of the system to the power supply. However, the information inside CMOS memories is volatile so they cannot be switched off completely. It has been reported that static random access memories (SRAMs) can keep the information even if the bias voltage falls down to $15-20 \%$ of the nominal value [1]. Very often, the combinational logic driving the memory cells does not work until the bias voltage returns to the nominal value, but the information does not disappear.

The problem is that, as the bias voltage decreases, the critical charge to trigger a single event upset (SEU) decreases as well. This is also valid for other single events, such as multiple cell/bit upsets (MCU, MBU), etc., with the possible exception of the single event latch-ups (SEL). This fact has led to the demonstration that even electrons, the lightest charged particles, provoke bitflips in 45-nm CMOS SRAMs at ultralow bias voltage [1].

Recently, a new kind of SRAM has drawn the attention of different research groups. These memories are built by Renesas Electronics in 150-nm CMOS technology and they are called Advanced Low Power SRAMs (A-LPSRAM) [2].

This work was supported in part by the Spanish MCINN project TIN201340968-P, by the Secretaría de Educación Superior Ciencia Tecnología e Innovación del Ecuador (SENESCYT), and by the "José Castillejo" mobility grant for professors and researchers.

J. A. Clemente and H. Mecha are with the Computer Architecture Department, Facultad de Informática, Universidad Complutense de Madrid (UCM), Spain, e-mail: ja.clemente, hmecha@fdi.ucm.es.

F. J. Franco and J. A. Agapito are with the Departamento de Física Aplicada III, Facultad de Ciencias Físicas, Universidad Complutense de Madrid (UCM), Spain, e-mail: fjfranco, agapito@fis.ucm.es.

R. Velazco is with the Université Grenoble-Alpes \& CNRS, TIMA, Grenoble (France), e-mail: raoul.velazco@imag.fr.

P. Ramos and V. Vargas are with the Université Grenoble-Alpes \& CNRS, TIMA, Grenoble (France), and with the Universidad de las Fuerzas Armadas, ESPE, DEEE, Sangolqui, Ecuador, e-mail: pframos, vcvargas@espe.edu.ec.

F. Villa and M. Baylac are with the Laboratoire de Physique Subatomique et de Cosmologie LPSC, Université Grenoble-Alpes \& CNRS/IN2P3, Grenoble, France, e-mail: baylac, francesca.villa@lpsc.in2p3.fr.
Their main characteristic is that the individual 6-transistor cells are not built in planar technology but in a 3D structure with advantages such as the minimization of the cell area, the incorporation of capacitors that make the occurrence of bitflips more difficult, and the removal of the parasitic pnpn structures prone to trigger latch-ups. To the authors' knowledge, the first independent tests on A-LPSRAMs under radiation were performed at the NASA GSFC to investigate heavy ion effects [3]. Recently, the behavior of these memories under very energetic protons has been explored in order to propose its use in the CERN LHC upgrade [4]. Also, the memories were tested under $15-\mathrm{MeV}$ neutrons at the GENEPI2 facility at nominal bias voltage [5].

However, the tolerance of these memories to radiation has not still been investigated in special circumstances, where single events are easier to occur. As it was previously said, bitflips are much more likely to occur when the circuit operates at ultra-low bias voltage, not far from the minimum power supply that allows retention of data. Also, it has been demonstrated that typical static tests underestimate the cross sections so dynamic tests are necessary [6]-[8] for a full understanding of the occurrence of single events.

This paper investigates the behavior of A-LPSRAMs at bias voltages little above the minimum value in static mode, and in dynamic mode. As the first family of tests issued very interesting results, this paper will be devoted to study these phenomena, including new clusters of bitflips and hard errors at low bias voltage.

\section{EXPERIMENTAL Setup}

Tests were carried out on one sample of an A-LPSRAM with a capacity of $8 \mathrm{Mbit}$ and configured as $1 \mathrm{M} \times 8$ bits. According to the manufacturer, the nominal bias voltage ranges from 2.7 to $3.6 \mathrm{~V}$. However, it was verified that even with a bias voltage of $0.60-0.65 \mathrm{~V}$, the information previously written was not lost. Besides, the combinational blocks were operational even with a bias voltage value at least as low as $1.9 \mathrm{~V}$, value below which the read-out system does not work.

\section{A. GENEPI2 neutron source}

GENEPI2 (GEnerator of NEutrons Pulsed and Intense) is a neutron facility located at the LPSC (Laboratoire de Physique Subatomique et Cosmologie) in Grenoble, France. Since 2013, this accelerator has been used to irradiate integrated circuits from different technologies [5], [9]. It is an electrostatic accelerator producing neutrons by impinging a deuteron beam 
Table I

TIME SEQUENCE OF THE IRRADIATIONS

\begin{tabular}{cccccc}
\hline Round & Pattern & Kind & $V_{C C}$ & Fluence & Affected addresses \\
\hline $\mathrm{A}$ & $0 \times 00$ & Stat. & 0.70 & 2.04 & 342 \\
$\mathrm{~B}$ & $0 \times 55$ & Stat. & 0.80 & 2.04 & 131 \\
$\mathrm{C}$ & $0 \times 55$ & Stat. & 0.90 & 2.04 & 1 \\
\hline $\mathrm{D}+\mathrm{E}$ & $0 \times 55$ & Stat. & 1.00 & 6.11 & 0 \\
$\mathrm{~F}$ & $0 \times 55$ & Stat. & 0.70 & 2.04 & 130 \\
$\mathrm{G}$ & $0 \times 55$ & Stat. & 0.75 & 2.05 & 338 \\
\hline $\mathrm{H}$ & $0 \times 55$ & Stat. & 0.85 & 2.04 & 312 \\
$\mathrm{I}$ & $N / A$ & Dyn. & 3.30 & 0.68 & bborted \\
$\mathrm{J}$ & $0 \times 55$ & Stat. & 0.90 & 4.07 & 5 \\
\hline $\mathrm{K}$ & $0 \times 55$ & Stat. & 0.85 & 2.04 & 129 \\
$\mathrm{~L}$ & $0 \times 55$ & Stat. & 0.80 & 2.05 & 132 \\
$\mathrm{M}$ & $0 \times 55$ & Stat. & 0.75 & 2.04 & 134 \\
\hline $\mathrm{N}$ & $0 \times 55$ & Stat. & 0.70 & 2.04 & 133 \\
$\mathrm{O}$ & $0 \times 55$ & Stat. & 0.95 & 6.11 & 2 \\
$\mathrm{P}$ & $N / A$ & Dyn. & 3.30 & 4.28 & 0 \\
$\mathrm{Q}$ & $N / A$ & Dyn. & 2.20 & 4.28 & 0 \\
\hline & & & $\mathrm{V}$ & $\times 10^{9} \mathrm{n} / \mathrm{cm}^{2}$ &
\end{tabular}

onto a fixed target. The target contains either Tritium (T) or Deuterium (D) according to the required neutron energy. After acceleration, deuterons (d) produce neutrons (n) by one of the following fusion reactions: $d+T \rightarrow n+H e^{4}$ and $d+D \rightarrow n+H e^{3}$.

Neutrons are produced with an average energy of either 14.2 $\mathrm{MeV}$ for the first reaction or $2.5 \mathrm{MeV}$ for the second reaction. The Devices Under Test (DUTs) are set facing directly the target at a distance determined to match the required neutron flux. Thus, only the most energetic neutrons $(15 \mathrm{MeV})$ hit the device.

Neutron production is monitored throughout the experiments to determine the neutron dose for each irradiation. Early 2015, a fresh tritium target was installed, generating a maximum neutron flux of $4.5 \times 10^{7} \mathrm{n} \cdot \mathrm{cm}^{-2} \cdot \mathrm{s}^{-1}$. Under these conditions, DUTs are exposed to a dose of $1.6 \times 10^{11}$ $n \cdot \mathrm{cm}^{-2}$ within one hour.

\section{B. Test system}

The memory was written and read by means of a PIC18F85J90 microcontroller. This device worked with a 20$\mathrm{MHz}$ quartz clock and, experimentally, it was observed that it could work even with a bias voltage of $1.9 \mathrm{~V}$.

In the low-bias voltage static tests, the microcontroller and the SRAM were biased by two independent power supplies. That of the microcontroller was fixed to $3.3 \mathrm{~V}$, and that of the SRAM was tunable from 0 to $3.3 \mathrm{~V}$. SRAM bias voltage was measured with a Keithley 2001 multimeter directly at the memory board. The three grounds (two for the power supplies, and one for the multimeter) were connected near the SRAM board for an accurate measurement. That means that six shielded cables, with a length of 20-m each, were necessary to build the electronic system.

In low-bias voltage static tests, address and data buses, as well as enable signals were set to ground in order to avoid the activation of over-voltage protection structures present in the memories. In consequence, a $0 \times 00$ word was always written in the first address in the memory, $0 \times 00000$, whichever the pattern was. Let us bear in mind that only 8 bits were sacrificed in a memory with $8 \mathrm{Mbits}$ and, also, it allows testing and debugging the routines to detect the bitflips.

\section{RESUlTS}

As shown in Table I, the tests were performed in successive steps. As there was not any hint to predict the behavior of the memories with ultra-low bias voltages, the irradiations were performed in long steps, next choosing intermediate values, and repeating voltage values at the end of the experiments.

One can see that, at very low voltage values, hundreds of addresses are affected, with single bitflips or MBUs. In general, errors in the irradiations fall in three categories: Clusters of bitflips, hard errors at low bias voltage, and SEUs.

\section{A. Static low bias voltage tests}

1) Clusters of bitflips: Irradiations with hundreds of bitflips are characterized by the occurrence of clusters of errors (rounds A-B, F-H, K-N). It is necessary that the bias voltage be $0.85 \mathrm{~V}$ or below. Words are corrupted as simple bitflips or events of diverse multiplicity in very close addresses. In particular, the distance between the lowest and highest address of each cluster is around 128. Even more, both boundaries are close to consecutive integer multiples of 128 . Table II shows the characteristics of the clusters observed with bias voltage of $0.85 \mathrm{~V}$. In one round, four clusters of different sizes and with error multiplicity up to 7 were registered starting in different addresses and finishing near the end of the address vector. In the other round, the cluster appeared at the beginning of the memory. This fact seems to be universal since clusters never appear in the middle of the memory.

2) Hard errors at low bias voltage: As the tests were performed, it was observed that some addresses reappeared from round to round. For example, it was observed that, from round $\mathrm{K}$ and below $0.90 \mathrm{~V}$, the word stored in $0 \times 4 D 69 C$, supposed to be $0 \times 55$, systematically changed into $0 \times D 5$, behavior not observed in the pristine memory. This phenomenon was observed in seven addresses of the memory (Table III). Once the irradiations ended, the memories were checked at the nominal voltage writing different patterns and reading the information, and these addresses seemed to work correctly. However, if the bias voltage falls to $0.7 \mathrm{~V}$, bitflips in the problematic addresses appear again.

A deeper analysis of the results of this table shows that the error in address \#6 was not detected during the irradiations since the checkerboard pattern, $0 \times 55$, did not change. Besides, errors in addresses \#2 and \#7 should have been detected in the tests but they were not. Probably, the bit was affected during the last rounds, when dynamic tests at nominal voltages were performed. More interestingly, when the memory was checked one week after the end of the tests, addresses \#3 and \#4 worked fine. This fact indicates the existence of some kind of physical annealing that mitigates the radiation damage.

These hard errors are roughly similar to the well-known stuck bits, in which the information inside the bit cannot be changed, with the difference that they only occur if there is a cycle of low bias voltage. 
Table III

ADDRESSES WITH CORRUPTED INFORMATION AT $0.7 \mathrm{~V}$ AND OFFLINE

\begin{tabular}{ccccc}
\hline Written Pattern & Address & Read Word & First Det. & Id. \\
\hline \multirow{4}{*}{$0 \times 00$} & $0 \times 4 D 69 C$ & $0 \times 80$ & Round K & $\# 1$ \\
& $0 \times 8 B A E 7$ & $0 \times 08$ & Offline & $\# 2$ \\
\cline { 2 - 5 } & $0 \times 9 C E 7 B$ & $0 \times 02$ & Round J & $\# 3$ \\
& $0 \times C 27 A C$ & $0 \times 80$ & Round M & $\# 4$ \\
& $0 \times E D D 7 F$ & $0 \times 80$ & Round L & $\# 5$ \\
\hline \multirow{2}{*}{$0 \times F F$} & $0 \times 31 B D 9$ & $0 \times F 7$ & Offline & $\# 6$ \\
& $0 \times 42 F E 3$ & $0 \times F E$ & Offline & $\# 7$ \\
\hline
\end{tabular}

3) Single event upsets: Once the bitflips appearing in clusters of errors and those present in different radiation rounds were removed, there was a set of bitflips with characteristics agreeing with those expected in single event upsets: Isolation, randomness, occurrence in only one round, etc. Curiously, this kind of events were not the main contribution to the whole set of errors. For instance, only 4 SEUs were detected combining the two rounds with $0 \times 55$ pattern at $0.7 \mathrm{~V}$, the worst-case situation.

Fig. 1 shows the dependence of the SEU cross section with the bias voltage. As the number of errors is not high, error bars are wide and little information can be obtained. At any rate, it seems that the cross section decreases for higher values of the bias voltage, as usually happens in other SRAMs. Above $1 \mathrm{~V}$, no SEUs were observed. Using the procedure explained in [5] and references therein, the conclusion is that the SEU cross section is below $7.2 \cdot 10^{-17} \mathrm{~cm}^{2} /$ bit for this kind of radiation at $V_{C C}=1 \mathrm{~V}$ with $95 \%$-confidence. This figure beats in more than one order of magnitude the first estimation, $2.0 \cdot 10^{-15}$ $\mathrm{cm}^{2} / \mathrm{bit}$ at $V_{C C}=3.3 \mathrm{~V}$, presented in a previous work [5].

\section{B. Dynamic tests}

Dynamic tests at $3.3 \mathrm{~V}$ and $2.2 \mathrm{~V}$ were carried out until reaching a fluence of $4.28 \times 10^{9} \mathrm{n} / \mathrm{cm}^{2}$. The latter value was used instead of the threshold one of $1.9 \mathrm{~V}$ in order to prevent the microcontroller from slowing down. Read and write operations were performed while the memory was irradiated. In particular, the MARCH-C algorithm was executed [8].

No errors were observed during these tests. As done in Section III-A3, the conclusion is that the cross section is below $1.0 \cdot 10^{-16} \mathrm{~cm}^{-2} / \mathrm{bit}$ for this kind of radiation.

\section{Discussion}

In a previous work, it was concluded that the SEU cross section in A-LPSRAM memories was two orders of magnitude

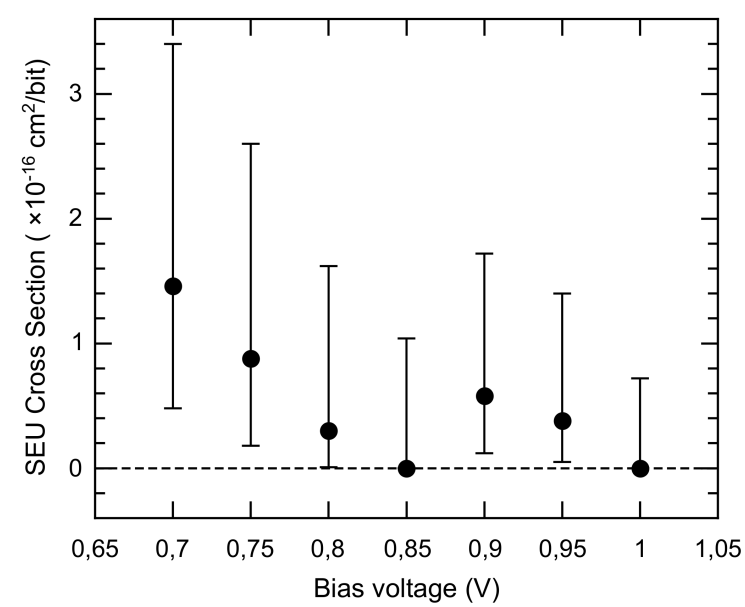

Figure 1. SEU cross section for the A-LPSRAM at ultra-low bias voltage. Error bars were calculated with $95 \%$-confidence.

below those of its counterparts in bulk CMOS technology [5]. However, as other authors have reported SEUs with protons and heavy ions [3], [4], it was likely that SEUs appeared at low values of bias voltage since there is evidence that, at least in bulk CMOS memories, the cross section exponentially grows as the bias voltage approaches the minimum value [10]. Our results show that, indeed, there is an increase in the cross section as the bias voltage gets closer to $0.65 \mathrm{~V}$ but, apparently, this growth is not as dramatical as in other memories. Thus, ALPSRAMs can be safely used with $1-\mathrm{V}$ bias voltage in systems exposed to neutron radiation.

Another interesting feature of this family of memories is the absence of errors in dynamic tests. As the system was controlled by a microcontroller with a $20-\mathrm{MHz}$ clock, it was not possible to make the SRAMs work near their speed limit as suggested in [7], [8]. However, other SRAMs in 90-nm bulk CMOS technology from another manufacturer showed thousands of events when they were tested in identical conditions to those of the A-LPSRAMs. This good robustness in the dynamic test can be attributed to the absence of physical structures prone to trigger microlatchups. As mentioned in Section I, one of the advantages of the 3D-structure of the A-LPSRAMs is the removal of pnpn paths where latch-ups (destructive or microlatchup) originate. According to [7], [8], events with tens of bitflips observed in dynamic tests appear after a microlatchup affecting one of the cell blocks into which the memory is divided. As A-LPSRAM are immune to microlatchups, this phenomenon cannot occur.

Table II

CHARACTERISTICS OF CLUSTERS OF BITFLIPS WITH $V_{C C}=0.85 \mathrm{~V}$

\begin{tabular}{|c|c|c|c|c|c|c|c|c|c|c|c|}
\hline \multirow{2}{*}{ Round } & \multirow{2}{*}{ Start } & \multirow{2}{*}{ End } & \multirow{2}{*}{ Affected addresses } & \multicolumn{8}{|c|}{ Multiplicity of the events } \\
\hline & & & & 1 & 2 & 3 & 4 & 5 & 6 & 7 & 8 \\
\hline $\mathrm{H}$ & $0 \times F 9980$ & $0 \times F 99 F F$ & 94 & 41 & 41 & 10 & 2 & 0 & 0 & 0 & 0 \\
\hline $\mathrm{H}$ & $0 \times F E 982$ & $0 \times F E 9 F F$ & 55 & 47 & 7 & 0 & 1 & 0 & 0 & 0 & 0 \\
\hline $\mathrm{H}$ & $0 \times F F 180$ & $0 \times F F 1 F C$ & 35 & 29 & 6 & 0 & 0 & 0 & 0 & 0 & 0 \\
\hline $\mathrm{H}$ & $0 \times F F F 80$ & $0 \times F F F F E$ & 127 & 2 & 9 & 27 & 34 & 31 & 20 & 4 & 0 \\
\hline $\mathrm{K}$ & $0 \times 00001$ & $0 \times 0007 F$ & 127 & 1 & 4 & 12 & 26 & 27 & 34 & 17 & 6 \\
\hline
\end{tabular}


Hard errors at low voltage share some properties with standard stuck bits. Like this well-known hard errors [11], those observed in the A-LPSRAMs vanish days or weeks after the irradiations, and whichever information is written, the bit ends up returning to a stable value. Nevertheless, differences are also evident and this avoids naming them as "stuck bits": Errors keep latent until a low bias-voltage cycle. Also, it is not possible to deduce from the results if the number of errors is proportional to the accumulated neutron fluence.

To the authors' opinion, the hard errors reported in this paper and classical stuck bits share a similar origin: microdose effects [12]. Indeed, it is easy to demonstrate that the CMOS inverters inside a SRAM cell only work if $V_{L}>V_{T H N}+$ $\left|V_{T H P}\right| ; V_{L}$ being the power supply value, and $V_{T H X}$ the threshold voltages of the transistors in the inverters. Typically, microdose effects occur with heavy ions but secondary ions generated after the collision of a neutron with silicon nuclei have identical ionizing properties. Therefore, if the threshold voltage of one of the transistors shifts, the minimum bias voltage for the affected cell locally grows. During the low bias voltage cycle, the affected cell is switched off, losing the stored information and when the bias voltage returns to the nominal value, the cell goes to the preferred state after powering up, that can be the same as the original written bit or not. In this last case, a bitflip would be observed.

Due to the absence of information about the way the memories are built, the mechanism leading to the appearance of clusters of bitflips is not fully understood. However, some properties cast light about this phenomenon. First of all, the omnipresence of 128 indicates that the memory may be organized in blocks of 128 8-bit words (1024 bits). Interleaving or error correction codes (ECC) seem to be absent in this memory due to two reasons: The addition of capacitors is a good shield against soft errors so it is nonsense to make the internal organization complicated. Besides, corrupted words in the clusters show events with a multiplicity of up to 8 , fact in contradiction with structures with interleaving.

Recent works have demonstrated that clusters of bitflips in bulk CMOS SRAMs are caused by the malfunction of the physical block where the cells are integrated [7], [8]. In this case, the phenomenon is the propagation of microlatch-ups only stopped by the borders of the block. However, in other technologies, latch-ups usually appear only at high values of bias voltage, exactly the opposite behavior observed in the A-LPSRAMs. A-LPSRAMs are supposed to be latchup-free; finally, it was not necessary to switch the memory off to make it working properly again.

One possibility is that, if every block is biased by its own driver, single event transients can lead to a spurious decrease in the bias voltage of the block that erases the stored information. This may explain why clusters do not occur above $0.85 \mathrm{~V}$ since even the largest transients would not reach the threshold value to delete the information in the cells. At any rate, other theories can be proposed. It is likely that the row \& column decoders play an important but not understood role in this phenomenon. In other memories from the same manufacturer in the range of several Mbits, the datasheets indicate that 7 address bits $\left(2^{7}=128\right)$ are used for the column decoder, leaving the rest for the row decoder. The abundance of clusters in the addresses between $0 \times 00000$ and $0 \times 000 E F$ and around $0 \times F F F F F$ could be related to the fact that the address bus needed to be set to $0 \times 00000$ in the experiments. Further work should be done to ellucidate the characteristics of this phenomenon.

\section{CONCLUSiOnS}

This paper has presented experimental evidences of the sensitivity to $14-\mathrm{MeV}$ neutrons of an 8 Mbit LPSRAM, manufactured by Renesas Electronics in 150-nm CMOS technology, when powered up at low bias voltage. Interesting conclusions can be drawn from the performed experiments. Results issued from radiation ground tests confirmed that the memory has a cross section below $7.2 \times 10^{-17} \mathrm{~cm}^{2} /$ bit with a $95 \%$-confidence above $1 \mathrm{~V}$. Below that voltage, new kinds of errors were identified: clusters of bitflips in groups of addresses which first and last elements were approximately never more than 128 positions away; and hard errors that were only visible at low voltage, even when the memory is no longer exposed to radiation.

\section{REFERENCES}

[1] M. P. King et al., "Electron-Induced Single-Event Upsets in Static Random Access Memory," IEEE Tran. Nucl. Sci., vol. 60, pp. 41224129, Dec. 2013.

[2] Renesas Electronics, "About LPSRAM Effort." [Online]. Available: http://www.renesas.eu/products/memory/low\_power_ssram/child/ renesas\_effort.jsp.

[3] M. O'Bryan et al., "Compendium of Recent Single Event Effects Results for Candidate Spacecraft Electronics for NASA," in IEEE Radiation Effects Data Workshop (REDW), pp. 11-20, July 2008.

[4] S. Uznanski et al., "The Effect of Proton Energy on SEU Cross Section of a 16 Mbit TFT PMOS SRAM with DRAM Capacitors," IEEE Tran. Nucl. Sci., vol. 61, pp. 3074-3079, Dec. 2014.

[5] R. Velazco et al., "Evidence of the Robustness of a COTS Soft-Error Free SRAM to Neutron Radiation," IEEE Tran. Nucl. Sci., vol. 61, pp. 3103-3108, Dec. 2014.

[6] G. Tsiligiannis et al., "Testing a Commercial MRAM Under Neutron and Alpha Radiation in Dynamic Mode," IEEE Tran. Nucl. Sci., vol. 60 , pp. 2617-2622, Aug. 2013.

[7] G. Tsiligiannis et al., "Multiple Cell Upset Classification in Commercial SRAMs," IEEE Tran. Nucl. Sci., vol. 61, pp. 1747-1754, Aug. 2014.

[8] G. Tsiligiannis et al., "Dynamic Test Methods for COTS SRAMs," IEEE Tran. Nucl. Sci., vol. 61, pp. 3095-3102, Dec. 2014.

[9] F. Villa et al., "Accelerator-Based Neutron Irradiation of Integrated Circuits at GENEPI2 (France)," in IEEE Radiation Effects Data Workshop (REDW), pp. 1-5, July 2014.

[10] A. Vazquez-Luque et al., "Neutron Induced Single Event Upset Dependence on Bias Voltage for CMOS SRAM With BPSG," IEEE Tran. Nucl. Sci., vol. 60, pp. 4692-4696, Dec. 2013.

[11] L. D. Edmonds et al., "Ion-induced stuck bits in 1T/1C SDRAM cells," IEEE Tran. Nucl. Sci., vol. 48, pp. 1925-1930, Dec. 2001.

[12] A. Haran et al., "Single Event Hard Errors in SRAM Under Heavy Ion Irradiation," IEEE Tran. Nucl. Sci., vol. 61, pp. 2702-2710, Oct. 2014. 